\title{
Could this be the tip of the iceberg? Endocrine dysfunction of immune checkpoint inhibitors at Kent Oncology Centre
}

\author{
T. Wang, S. Anandappa, S. Sivappriyan, J. Kumar \\ Endocrinology Department, Maidstone Hospital
}

Category: Neoplasia, cancer \& late effects

INTRODUCTION

- Immune checkpoint inhibitors generate immune responses to tumours, causing them to be rejected, and break tumour-induced immune tolerance. Used for advanced neoplasias (incl. metastatic melanoma, non-small-cell lung cancer, and advanced renal-cell carcinoma), their mechanism of action can lead to a variety of inflammatory toxicities.

- Baseline clinical and biochemical endocrine assessment at the start of immune checkpoint treatment and each treatment cycle ${ }^{1}$ is important given the treatable nature of it.

- The improvements in survival of these patients necessitate further long-term screening.

- Our study was to look at various aspects of this screening with a view to improve our knowledge and also patient care.

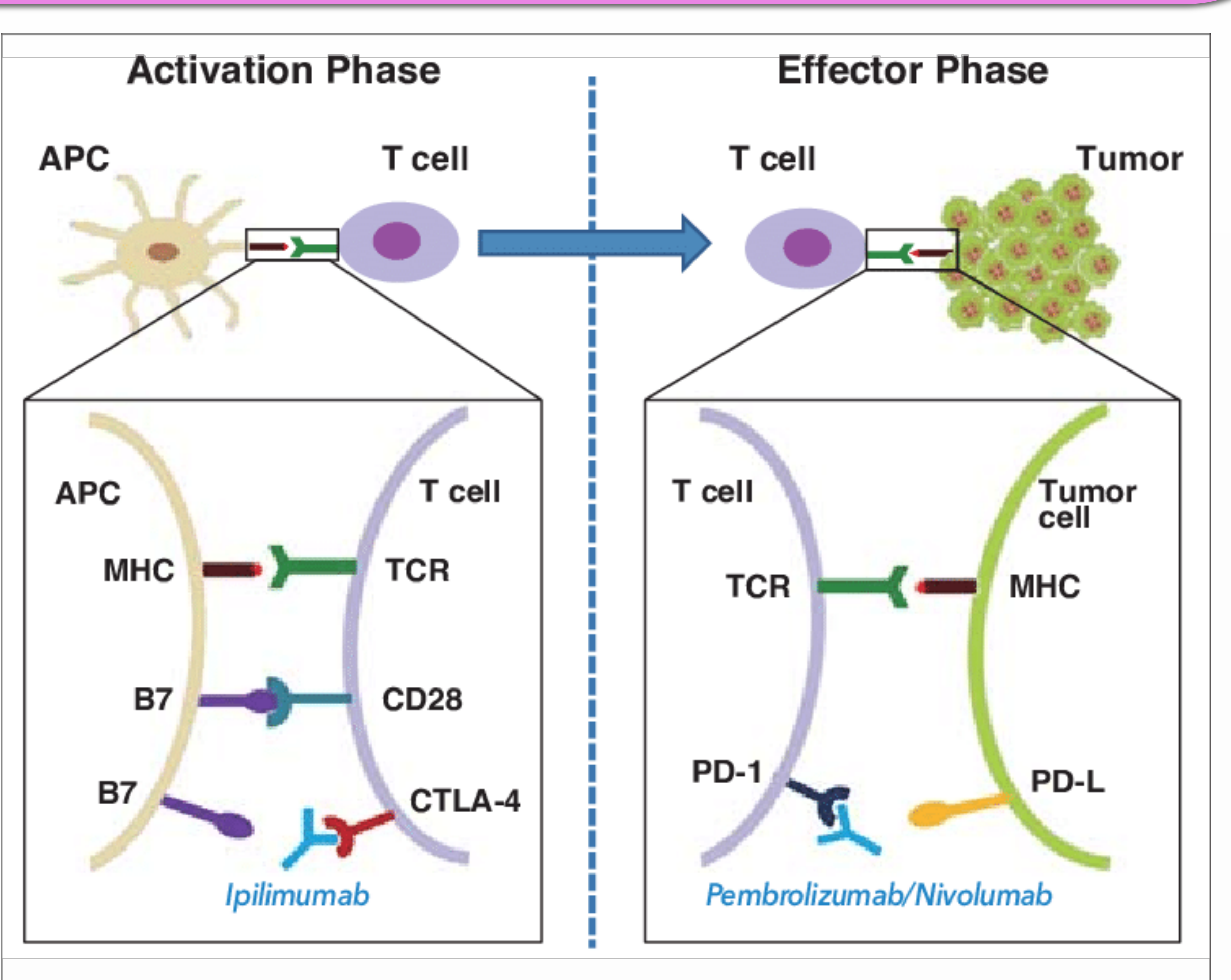

\section{METHODS}

- Patients receiving Ipilimumab \&/or Pembrolizumab between 01/01/2016 and 30/09/2016 at Kent Oncology Centre were identified using ARIA prescribing system.

- Data for 31 patients were collected using using Telepath, PACS, KOMS \& Allscript letter records and analysed using Microsoft Excel.

- 1 patient excluded due to lack of access to their blood results.

- Reviewed to see if baseline endocrine tests (TSH, FT4, 9 am cortisol, pituitary functions +/- imaging) then subsequently 3 weekly thyroid function tests were carried out as per local guidelines; and the outcomes of these results for 39 weeks following administration of the immune checkpoint inhibitor.

\section{RESULTS}

- 31 patients: 17 Males and 14 Females.

- No full set of baseline endocrine tests done.

- 7 out of 31 patients had appropriate TSH monitoring throughout their treatment.

- $\mathbf{2 5 . 8 1 \%}$ developed endocrine complications following immunotherapy.

- Onset varied between 3 weeks and 36 weeks after commencing treatment.

- 2 out of 8 patients who developed an endocrine abnormality were referred to an endocrine team.

- 1 out of 8 patients had a full endocrine profile done after an abnormality was detected.

- None of the patients with an endocrine abnormality underwent pituitary imaging.

\section{CONCLUSION}

- A high prevalence of endocrine dysfunction indicates the need for collaboration between oncologists and endocrinologists

- Robust guidelines to be adhered to when prescribing an immune checkpoint inhibitor. If abnormalities are detected, a full pituitary screening (biochemistry and imaging) should be undertaken.

- The follow up period for endocrine function after administration of immune checkpoint inhibitors needs to be extended globally to at least 36 weeks
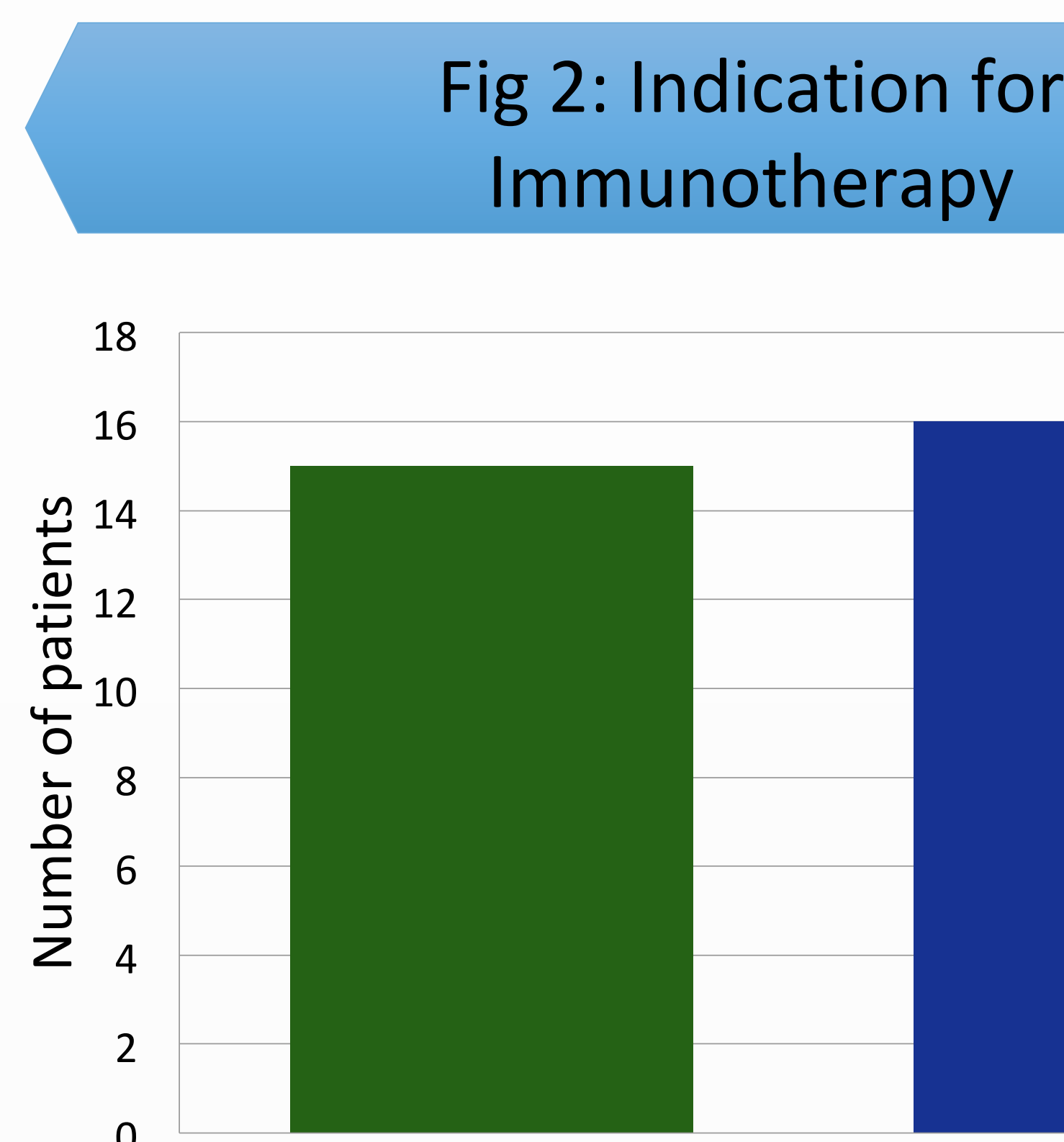

Immunotherapy

Malignant melanoma Non small cell lung

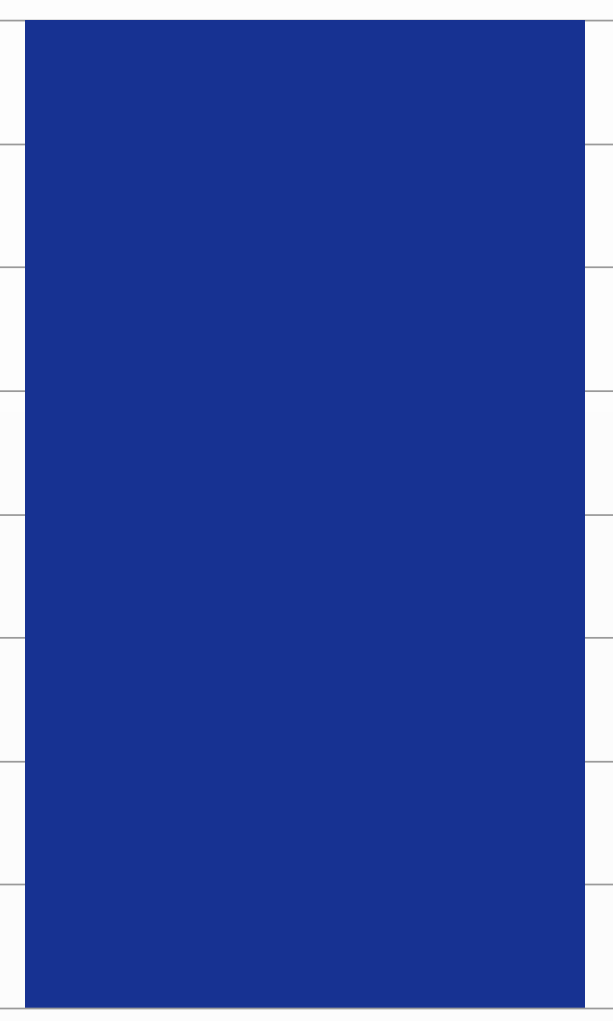

Diagnosis

Fig 3: Type of Immunotherapy used

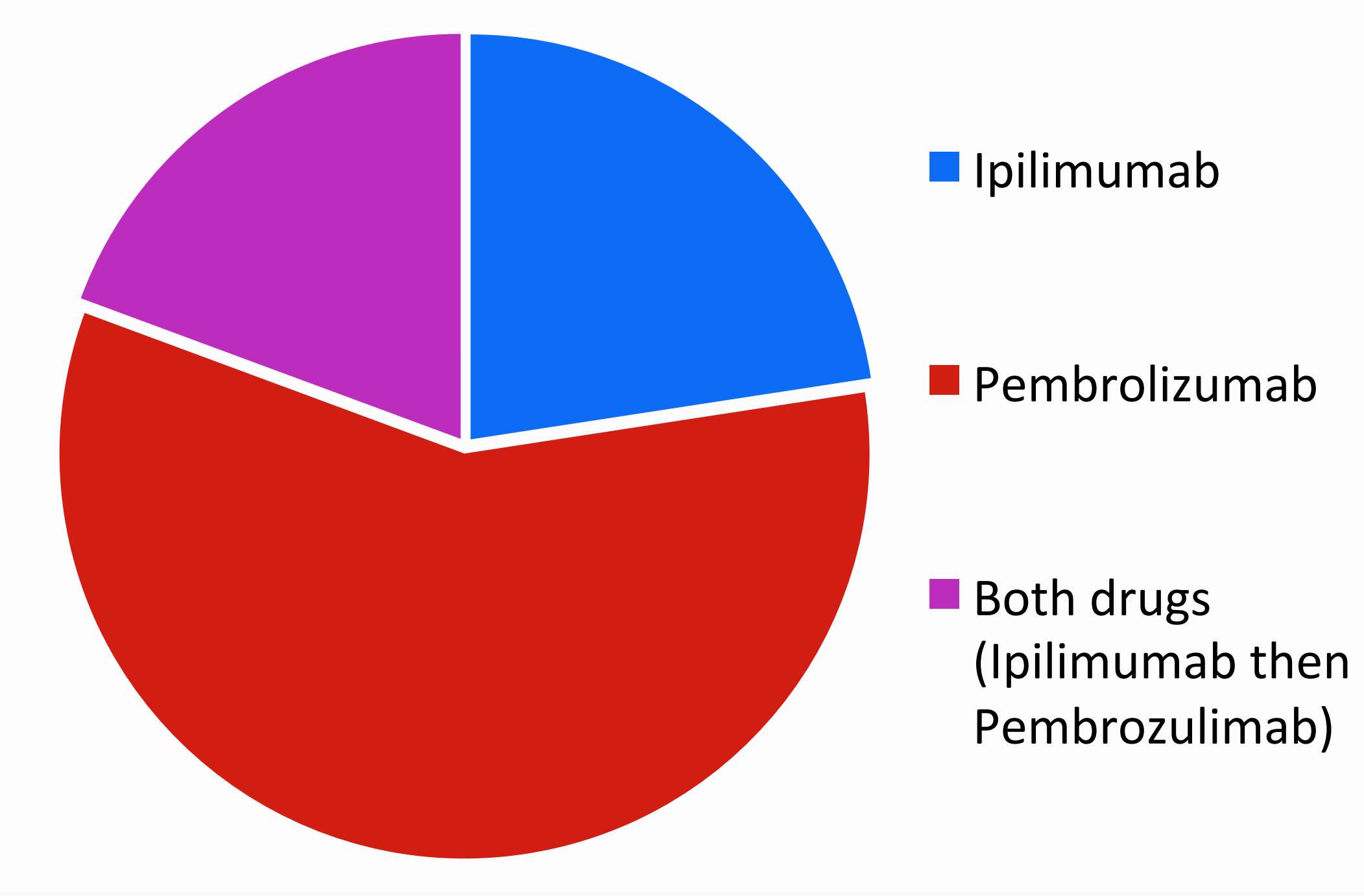
- Non small cell lung cancer patients were treated with Pembrolizumab only.
Fig 4: Types of endocrine complications

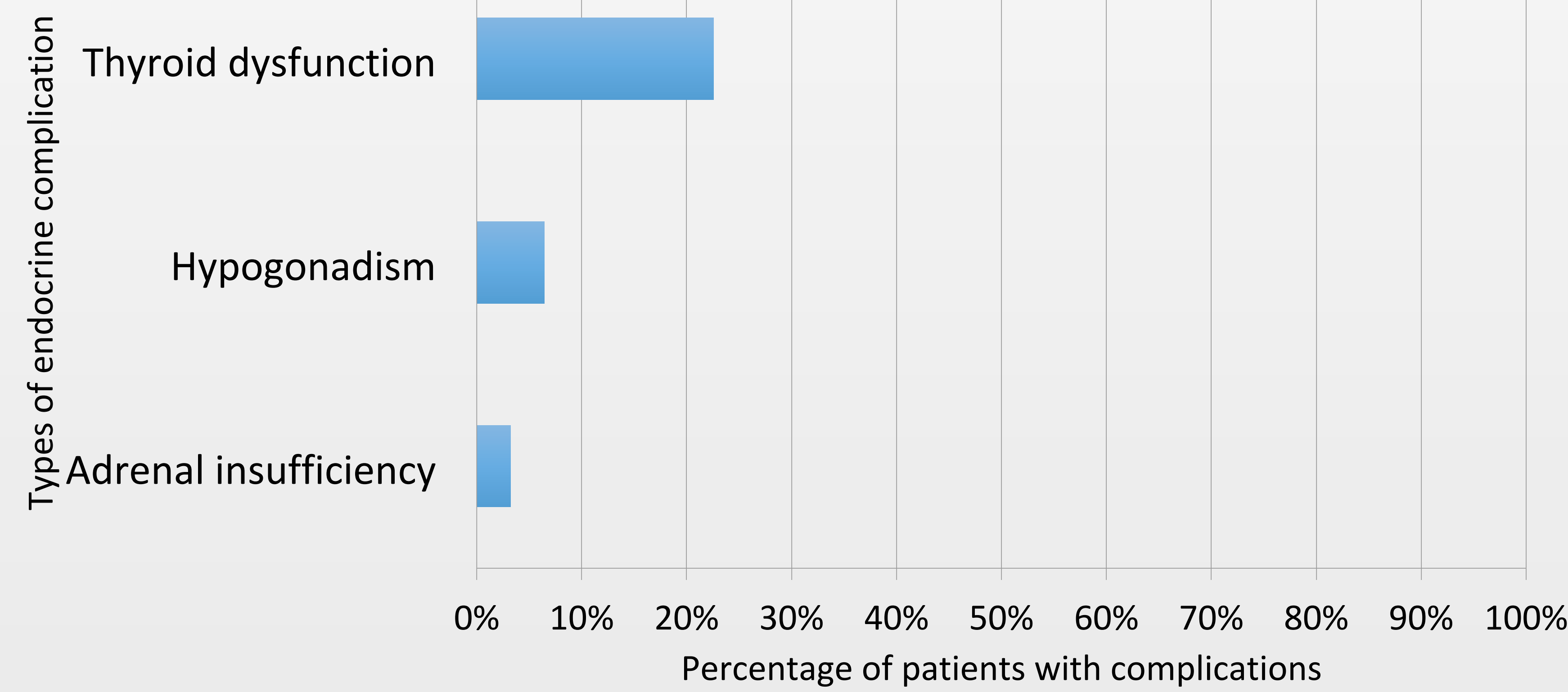

Fig 5: Percentage of patients experienced endocrine dysfunction in following immune checkpoint inhibitor/s

REFERENCES

1) Joshi et al, 13/04/2016; Immune checkpoint inhibitor-related hypophysitis and endocrine dysfunction: clinical review; http://onlinelibrary.wiley.com/doi/10.1111/cen.13063/ful

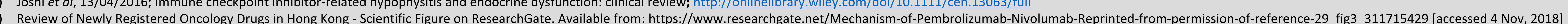

